\title{
CHAPTER IV
}

\section{RESOLUTIONS OF THE GENERAL ASSEMBLY}

\section{Report of the Resolutions Committee}

Membership: J. Kovalevsky, Y. Kozai, D. McNally (Chairman), V. Trimble, P. Wayman. Sadly before the Committee could begin its work, Patrick Wayman died. He was not replaced on the Committee.

1. The Committee communicated by mail and e-mail prior to the General Assembly, held one final meeting, and several informal meetings, between the two sessions of the General Assembly.

2. The Committee received no Resolutions from the Executive Committee or from the Adhering Organisations. No Resolutions were received which had financial implications for the Union. Five scientific Resolutions of Type B were received, one of which was inadmissable and a second was withdrawn consequent upon discussion between the two sessions of the General Assembly.

D. McNally 


\title{
APPROVED RESOLUTIONS/RESOLUTIONS APPROUVEES
}

\section{B1.1 MAINTENANCE AND ESTABLISHMENT OF REFERENCE FRAMES AND SYSTEMS}

\author{
The XXIVth International Astronomical Union General Assembly,
}

\section{Noting}

1. that Resolution B2 of the XXIIIrd General Assembly (1997) specifies that "the fundamental reference frame shall be the International Celestial Reference Frame (ICRF) constructed by the IAU Working Group on Reference Frames",

2. that Resolution B2 of the XXIIIrd General Assembly (1997) specifies "That the Hipparcos Catalogue shall be the primary realisation of the International Celestial Reference System (ICRS) at optical wavelengths", and

3. the need for accurate definition of reference systems brought about by unprecedented precision, and

\section{Recognising}

1. the importance of continuing operational observations made with Very Long Baseline Interferometry (VLBI) to maintain the ICRF,

2. the importance of VLBI observations to the operational determination of the parameters needed to specify the time-variable transformation between the International Celestial and Terrestrial Reference Frames,

3. the progressive shift between the Hipparcos frame and the ICRF, and

4. the need to maintain the optical realisation as close as possible to the ICRF,

\section{Recommends}

1. that IAU Division I maintain the Working Group on Celestial Reference Systems formed from Division I members to consult with the International Earth Rotation Service (IERS) regarding the maintenance of the ICRS,

2. that the IAU recognise the International VLBI service (IVS) for Geodesy and Astrometry as an IAU Service Organisation,

3. that an official representative of the IVS be invited to participate in the IAU Working Group on Celestial Reference Systems, 
4. that the IAU continue to provide an official representative to the IVS Directing Board,

5. that the astrometric and geodetic VLBI observing programs consider the requirements for maintenance of the ICRF and linking to the Hipparcos optical frame in the selection of sources to be observed (with emphasis on the Southern Hemisphere), design of observing networks, and the distribution of data, and

6. that the scientific community continue with high priority ground- and space- based observations (a) for the maintenance of the optical Hipparcos frame and frames at other wavelengths and (b) for links of the frames to the ICRF.

\section{B1.1 SUIVI ET CONSTRUCTION DE REPERES ET DE SYSTEMES DE REFERENCE}

La XXIVème Assemblée générale de l'Union Astronomique Internationale,

\section{Notant}

1. que la résolution B2 de la XXIIlème Assemblée générale (1997) a spécifié que "le repère de référence fondamental sera le repère céleste international de référence (ICRF) construit par le groupe de travail de l'UAI sur les repères de référence",

2. que la résolution $B 2$ de la XXIIlème Assemblée générale (1997) a spécifié que "le catalogue Hipparcos sera la réalisation primaire du système céleste international de référence (ICRS) pour les longueurs d'onde optiques", et

3. la nécessité d'établir une définition exacte des systèmes de référence par suite d'une amélioration sans précédent de la précision des observations, et

\section{Reconnaissant}

1. l'importance de la poursuite des observations opérationnelles à l'aide de l'interférométrie à très longue base (VLBI) pour maintenir l'ICRF,

2. l'importance des observations VLBI pour une détermination opérationnelle des paramètres nécessaires pour déterminer la transformation dépendant du temps entre les repères internationaux de référence céleste et terrestre,

3. le décalage progressif entre le repère Hipparcos et l'ICRF, et

4. la nécessité de maintenir la réalisation optique aussi proche que possible de l'ICRF,

\section{Recommande}

1. que la Division I conserve le Groupe de travail sur les systèmes de référence célestes constitué de membres de la Division I comme consultant auprès du Service international de la rotation terrestre (IERS) en ce qui concerne la maintenance de l'ICRS, 
2. que l'UAI reconnaisse le Service international VLBI pour la géodésie et l'astrométrie (IVS) comme un organisme de service de l'UAI,

3. qu'un représentant officiel de l'IVS soit invité à participer au groupe de travail de l'UAI sur les systèmes de référence,

4. que l'UAI continue à désigner un représentant officiel au comité de direction de l'IVS,

5. que les programmes d'observation VLBI pour l'astrométrie et la géodésie prennent en compte dans la sélection des sources à observer, dans la conception des réseaux d'observation et dans la distribution des données (en particulier dans l'hémisphère sud), les besoins pour la maintenance de l'ICRF et pour la liaison avec le repère optique Hipparcos et,

6. que la communauté scientifique continue à effectuer, avec une grande priorité, des observations au sol et dans l'espace (a) pour la maintenance du repère Hipparcos ou à d'autres longueurs d'onde et (b) pour le raccordement de ces repères avec l'ICRF.

\section{B1.2 HIPPARCOS CELESTIAL REFERENCE FRAME}

The XXIVth International Astronomical Union General Assembly,

\section{Noting}

1. that Resolution B2 of the XXIIIrd General Assembly (1997) specifies, "That the Hipparcos Catalogue shall be the primary realisation of the International Celestial Reference System (ICRS) at optical wavelengths",

2. the need for this realisation to be of the highest precision,

3. that the proper motions of many of the Hipparcos stars known, or suspected, to be multiple are adversely affected by uncorrected orbital motion,

4. the extensive use of the Hipparcos Catalogue as reference for the ICRS in extension to fainter stars,

5. the need to avoid confusion between the International Celestial Reference Frame (ICRF) and the Hipparcos frame, and

6. the progressive shift between the Hipparcos frame and the ICRF,

\section{Recommends}

1. that Resolution B2 of the XXIIIrd IAU General Assembly (1997) be amended by excluding from the optical realisation of the ICRS all stars flagged C, G, O, V and X in the Hipparcos Catalogue, and

2. that this modified Hipparcos frame be labelled the Hipparcos Celestial Reference Frame (HCRF). 


\section{B1.2 LE REPERE DE REFERENCE CELESTE HIPPARCOS}

La XXIVème Assemblée générale de l'Union Astronomique Internationale,

\section{Notant}

1. que la résolution B2 de la XXIIIème Assemblée générale (1997) a spécifié que "le catalogue Hipparcos sera la réalisation primaire du système céleste international de référence (ICRS) pour les longueurs d'onde optiques",

2. la nécessité que cette réalisation ait la précision la plus grande possible,

3. que les mouvements propres de nombreuses étoiles Hipparcos connues ou suspectées d'être multiples sont affectés de manière défavorable par des mouvements orbitaux non corrigés,

4. l'usage extensif du catalogue Hipparcos comme référence à l'ICRS dans les travaux d'extension à des étoiles plus faibles,

5. la nécessité d'éviter toute confusion entre le repère céleste international de référence (ICRF) et le repère Hipparcos, et

6. le décalage progressif entre le repère Hipparcos et l'ICRF,

\section{Recommande}

1. que la résolution B2 de la XXIIlème Assemblée générale (1997) de l'UAI soit amendée en excluant de la réalisation optique de l'ICRS toutes les étoiles notées $\mathrm{C}, \mathrm{G}$, $\mathrm{O}$, $\mathrm{V}$ et $\mathrm{X}$ dans le catalogue Hipparcos, et (HCRF).

2. que ce repère Hipparcos modifié soit dénommé Repère de référence céleste Hipparcos

\section{B1.3 DEFINITION OF BARYCENTRIC CELESTIAL REFERENCE SYSTEM AND GEOCENTRIC CELESTIAL REFERENCE SYSTEM}

The XXIVth International Astronomical Union General Assembly,

\section{Considering}

1. that the Resolution A4 of the XXIst General Assembly (1991) has defined a system of space-time coordinates for (a) the solar system (now called the Barycentric Celestial Reference System, (BCRS)) and (b) the Earth (now called the Geocentric Celestial Reference System (GCRS)), within the framework of General Relativity,

2. the desire to write the metric tensors both in the BCRS and in the GCRS in a compact and self-consistent form,

3. the fact that considerable work in General Relativity has been done using the harmonic gauge that was found to be a useful and simplifying gauge for many kinds of applications, 


\section{Recommends}

1. the choice of harmonic coordinates both for the barycentric and for the geocentric reference systems,

2. writing the time-time component and the space-space component of the barycentric metric $g_{\mu \nu}$ with barycentric coordinates $(t, \mathbf{x})(t=$ Barycentric Coordinate Time (TCB)) with a single scalar potential $w(t, \mathbf{x})$ that generalises the Newtonian potential, and the space-time component with a vector potential $w^{i}(t, \mathbf{x})$; as a boundary condition it is assumed that these two potentials vanish far from the solar system,

explicitly,

$$
\begin{gathered}
g_{00}=-1+\frac{2 w}{c^{2}}-\frac{2 w^{2}}{c^{4}} \\
g_{0 i}=-\frac{4}{c^{3}} w^{i} \\
g_{i j}=\delta_{i j}\left(1+\frac{2}{c^{2}} w\right)
\end{gathered}
$$

with

$$
\begin{gathered}
w(t, \mathbf{x})=G \int d^{3} \mathbf{x}^{\prime} \frac{\sigma\left(t, \mathbf{x}^{\prime}\right)}{\left|\mathbf{x}-\mathbf{x}^{\prime}\right|}+\frac{1}{2 c^{2}} G \frac{\partial^{2}}{\partial t^{2}} \int d^{3} \mathbf{x}^{\prime} \sigma\left(t, \mathbf{x}^{\prime}\right)\left|\mathbf{x}-\mathbf{x}^{\prime}\right| \\
w^{i}(t, \mathbf{x})=G \int d^{3} x^{\prime} \frac{\sigma^{i}\left(t, \mathbf{x}^{\prime}\right)}{\left|\mathbf{x}-\mathbf{x}^{\prime}\right|} .
\end{gathered}
$$

here, $\sigma$ and $\sigma^{i}$ are the gravitational mass and current densities respectively,

3. writing the geocentric metric tensor $G_{a b}$ with geocentric coordinates $(T, \mathbf{X})(T=$ Geocentric Coordinate Time (TCG)) in the same form as the barycentric one but with potentials $W(T, \mathbf{X})$ and $W^{a}(T, \mathbf{X})$; these geocentric potentials should be split into two parts potentials $W_{E}$ and $W_{E}^{a}$ arising from the gravitational action of the Earth and external parts $W_{e x t}$ and $W_{e x t}^{a}$ due to tidal and inertial effects; the external parts of the metric potentials are assumed to vanish at the geocenter and admit an expansion into positive powers of $\mathbf{X}$,

explicitly,

$$
\begin{gathered}
G_{00}=-1+\frac{2 W}{c^{2}}-\frac{2 W^{2}}{c^{4}} \\
G_{0 a}=-\frac{4}{c^{3}} W^{a} \\
G_{a b}=\delta_{a b}\left(1+\frac{2}{c^{2}} W\right)
\end{gathered}
$$

the potentials $W$ and $W^{a}$ should be split according to

$$
\begin{aligned}
W(T, \mathbf{X}) & =W_{E}(T, \mathbf{X})+W_{e x t}(T, \mathbf{X}) \\
W^{a}(T, \mathbf{X}) & =W_{E}^{a}(T, \mathbf{X})+W_{e x t}^{a}(T, \mathbf{X})
\end{aligned}
$$

the Earth's potentials $W_{E}$ and $W_{E}^{a}$ are defined in the same way as $w$ and $w^{i}$ but with quantities calculated in the GCRS with integrals taken over the whole Earth, 
4. using, if accuracy requires, the full post-Newtonian coordinate transformation between the BCRS and the GCRS as induced by the form of the corresponding metric tensors,

explicitly, for the kinematically non-rotating GCRS ( $T=\mathrm{TCG}, t=\mathrm{TCB}, r_{E}^{i}=x^{i}-x_{E}^{i}(t)$, and a summation from 1 to 3 over equal indices is implied),

$$
\begin{gathered}
T=t-\frac{1}{c^{2}}\left[A(t)+v_{E}^{i} r_{E}^{i}\right]+\frac{1}{c^{4}}\left[B(t)+B^{i}(t) r_{E}^{i}+B^{i j}(t) r_{E}^{i} r_{E}^{j}+C(t, \mathbf{x})\right]+O\left(c^{-5}\right) \\
X^{a}=\delta_{a i}\left[r_{E}^{i}+\frac{1}{c^{2}}\left(\frac{1}{2} v_{E}^{i} v_{E}^{j} r_{E}^{j}+w_{e x t}\left(\mathbf{x}_{E}\right) r_{E}^{i}+r_{E}^{i} a_{E}^{j} r_{E}^{j}-\frac{1}{2} a_{E}^{i} r_{E}^{2}\right)\right]+O\left(c^{-4}\right)
\end{gathered}
$$

where

$$
\begin{gathered}
\frac{d}{d t} A(t)=\frac{1}{2} v_{E}^{2}+w_{e x t}\left(\mathbf{x}_{E}\right) \\
\frac{d}{d t} B(t)=-\frac{1}{8} v_{E}^{4}-\frac{3}{2} v_{E}^{2} w_{e x t}\left(\mathbf{x}_{E}\right)+4 v_{E}^{i} w_{e x t}^{i}\left(\mathbf{x}_{E}\right)+\frac{1}{2} w_{e x t}^{2}\left(\mathbf{x}_{E}\right) \\
B^{i}(t)=-\frac{1}{2} v_{E}^{i} v_{E}^{2}+4 w_{e x t}^{i}\left(\mathbf{x}_{E}\right)-3 v_{E}^{i} w_{e x t}\left(\mathbf{x}_{E}\right) \\
B^{i j}(t)=-v_{E}^{i} \delta_{a j} Q^{a}+2 \frac{\partial}{\partial x^{j}} w_{e x t}^{i}\left(\mathbf{x}_{E}\right)-v_{E}^{i} \frac{\partial}{\partial x^{j}} w_{e x t}\left(\mathbf{x}_{E}\right)+\frac{1}{2} \delta_{i j} \dot{w}_{e x t}\left(\mathbf{x}_{E}\right) \\
C(t, \mathbf{x})=-\frac{1}{10} r_{E}^{2}\left(\dot{a}_{E}^{i} r_{E}^{i}\right),
\end{gathered}
$$

here $x_{E}^{i}, v_{E}^{i}$, and $a_{E}^{i}$ are the components of the barycentric position, velocity and acceleration vectors of the Earth, the dot stands for the total derivative with respect to $t$, and

$$
Q^{a}=\delta_{a i}\left[\frac{\partial}{\partial x^{i}} w_{e x t}\left(\mathbf{x}_{E}\right)-a_{E}^{i}\right] .
$$

The external potentials, $w_{e x t}$ and $w_{e x t}^{i}$, are given by

$$
w_{e x t}=\sum_{A \neq E} w_{A}, \quad w_{e x t}^{i}=\sum_{A \neq E} w_{A}^{i},
$$

where $E$ stands for the Earth and $w_{A}$ and $w_{A}^{i}$ are determined by the expressions for $w$ and $w^{i}$ with integrals taken over body $\mathrm{A}$ only.

\section{Notes}

It is to be understood that these expressions for $w$ and $w^{i}$ give $g_{00}$ correct up to $O\left(c^{-5}\right)$, $g_{0 i}$ up to $O\left(c^{-5}\right)$, and $g_{i j}$ up to $O\left(c^{-4}\right)$. The densities $\sigma$ and $\sigma^{i}$ are determined by the components of the energy momentum tensor of the matter composing the solar system bodies as given in the references. Accuracies for $G_{a b}$ in terms of $c^{-n}$ correspond to those of $g_{\mu \nu}$.

the external potentials $W_{e x t}$ and $W_{e x t}^{a}$ can be written in the form

$$
\begin{aligned}
& W_{\text {ext }}=W_{\text {tidal }}+W_{\text {iner }}, \\
& W_{\text {ext }}^{a}=W_{\text {tidal }}^{a}+W_{\text {iner }}^{a} .
\end{aligned}
$$

$W_{\text {tidal }}$ generalises the Newtonian expression for the tidal potential. Post-Newtonian expressions for $W_{\text {tidal }}$ and $W_{\text {tidal }}^{a}$ can be found in the references. The potentials $W_{\text {iner }}$, $W_{i n e r}^{a}$ are inertial contributions that are linear in $\mathrm{X}^{a}$. The former is determined mainly by 
the coupling of the Earth's nonsphericity to the external potential. In the kinematically nonrotating Geocentric Celestial Reference System, $W_{i n e r}^{a}$ describes the Coriolis force induced mainly by geodetic precession.

Finally, the local gravitational potentials $W_{E}$ and $W_{E}^{a}$ of the Earth are related to the barycentric gravitational potentials $w_{E}$ and $w_{E}^{i}$ by

$$
\begin{gathered}
W_{E}(T, \mathbf{X})=w_{E}(t, \mathbf{x})\left(1+\frac{2}{c^{2}} v_{E}^{2}\right)-\frac{4}{c^{2}} v_{E}^{i} w_{E}(t, \mathbf{x})+O\left(c^{-4}\right), \\
W_{E}^{a}(T, \mathbf{X})=\delta_{a i}\left(w_{E}^{i}(t, \mathbf{x})-v_{E}^{i} w_{E}(t, \mathbf{x})\right)+O\left(c^{-2}\right) .
\end{gathered}
$$

\section{References}

Brumberg, V.A., Kopeikin, S.M., 1988, Nuovo Cimento B, 103, 63.

Brumberg, V.A., 1991, Essential Relativistic Celestial Mechanics, Hilger, Bristol.

Damour, T., Soffel, M., Xu, C., Phys.Rev. D, 43, 3273 (1991); 45, 1017 (1992); 47, 3124 (1993); 49, 618 (1994).

Klioner, S. A., Voinov, A.V., 1993, Phys Rev. D, 48, 1451.

Kopeikin, S.M., 1989, Celest. Mech. 44, 87.

\section{B1.3. DEFINITION DU SYSTEME DE REFERENCE CELESTE BARYCENTRIQUE ET DU SYSTEME DE REFERENCE CELESTE GEOCENTRIQUE}

La XXIVème Assemblée générale de l’Union Astronomique Internationale,

\section{Considérant}

1. que la résolution A4 de la XXIème Assemblée générale (1991) de l'UAI a défini un système de coordonnées d'espace-temps pour (a) le système solaire (appelé maintenant système de référence céleste barycentrique, BCRS) et (b) pour la Terre (appelé maintenant système de référence céleste géocentrique, GCRS), dans le cadre de la relativité générale,

2. qu'il est souhaitable d'écrire les tenseurs métriques aussi bien dans le BCRS que dans le GCRS sous une forme compacte et cohérente,

3. le fait qu'un travail considérable a été effectué en relativité générale en utilisant la jauge harmonique dont on a trouvé qu'elle était utile et permettait des simplifications dans de nombreux types d'applications,

\section{Recommande}

1. de choisir les coordonnées harmoniques à la fois pour les systèmes de référence barycentrique et géocentrique,

2. d'écrire les composantes temps-temps et espace-espace de la métrique barycentrique $g_{\mu \nu}$ avec les coordonnées barycentriques $(t, \mathbf{x})(t=$ Temps-coordonnée barycentrique (TCB)) en utilisant un seul potentiel scalaire $w(t, \mathbf{x})$ qui généralise le potentiel newtonien, et la composante espace-temps en utilisant le potentiel vecteur $w^{i}(t, \mathbf{x})$; on supposera, comme condition aux limites que ces deux potentiels s'annulent loin du Système solaire; 
de façon explicite,

$$
\begin{gathered}
g_{00}=-1+\frac{2 w}{c^{2}}-\frac{2 w^{2}}{c^{4}} \\
g_{0 i}=-\frac{4}{c^{3}} w^{i} \\
g_{i j}=\delta_{i j}\left(1+\frac{2}{c^{2}} w\right)
\end{gathered}
$$

avec

$$
\begin{gathered}
w(t, \mathbf{x})=G \int d^{3} \mathbf{x}^{\prime} \frac{\sigma\left(t, \mathbf{x}^{\prime}\right)}{\left|\mathbf{x}-\mathbf{x}^{\prime}\right|}+\frac{1}{2 c^{2}} G \frac{\partial^{2}}{\partial t^{2}} \int d^{3} \mathbf{x}^{\prime} \sigma\left(t, \mathbf{x}^{\prime}\right)\left|\mathbf{x}-\mathbf{x}^{\prime}\right| \\
w^{i}(t, \mathbf{x})=G \int d^{3} \mathbf{x}^{\prime} \frac{\sigma^{i}\left(t, \mathbf{x}^{\prime}\right)}{\left|\mathbf{x}-\mathbf{x}^{\prime}\right|}
\end{gathered}
$$

les quantités $\sigma$ et $\sigma^{i}$ sont respectivement la densité de masse gravitationnelle et la densité d'impulsion gravitationnelle,

3. d'écrire le tenseur métrique géocentrique $G_{a b}$ avec les coordonnées géocentriques $(T, \mathbf{X})(T=$ Temps-coordonnée géocentrique (TCG)) sous la même forme que pour le cas barycentrique, mais avec les potentiels $W(T, \mathbf{X})$ et $W^{a}(T, \mathbf{X})$; ces potentiels géocentriques devraient être scindés en deux parties : les potentiels $W_{E}$ et $W_{E}^{a}$ provenant de l'action gravitationnelle de la Terre et les potentiels extérieurs $W_{e x t}$ and $W_{e x t}^{a}$ dus aux marées et aux effets inertiels; on supposera que les parties externes des potentiels métriques s'annulent au géocentre et admettent un développement en puissances positives de $\mathbf{X}$;

de façon explicite,

$$
\begin{gathered}
G_{00}=-1+\frac{2 W}{c^{2}}-\frac{2 W^{2}}{c^{4}}, \\
G_{0 a}=-\frac{4}{c^{3}} W^{a} \\
G_{a b}=\delta_{a b}\left(1+\frac{2}{c^{2}} W\right),
\end{gathered}
$$

les potentiels $W$ et $W^{a}$ doivent être scindés selon

$$
\begin{gathered}
W(T, \mathbf{X})=W_{E}(T, \mathbf{X})+W_{e x t}(T, \mathbf{X}) \\
W^{a}(T, \mathbf{X})=W_{E}^{a}(T, \mathbf{X})+W_{e x t}^{a}(T, \mathbf{X})
\end{gathered}
$$

les potentiels terrestres $W_{E}$ et $W_{E}^{a}$ sont définis de la même manière que $w$ et $w^{i}$ mais avec des quantités calculées dans le GCRS avec les intégrales calculées sur l'ensemble de la Terre,

4. si l'exactitude recherchée le nécessite, on utilisera la transformation post-newtonienne des coordonnées entre le BCRS et le GCRS telle qu'elle est déduite de la forme des tenseurs métriques correspondants;

de façon explicite, pour le GCRS cinématiquement non tournant, ( $T=\mathrm{TCG}, t=\mathrm{TCB}$, $r_{E}^{i}=x^{i}-x_{E}^{i}(t)$ et avec la convention implicite de sommation de 1 à 3 sur les indices identiques), 


$$
\begin{gathered}
T=t-\frac{1}{c^{2}}\left[A(t)+v_{E}^{i} r_{E}^{i}\right]+\frac{1}{c^{4}}\left[B(t)+B^{i}(t) r_{E}^{i}+B^{i j}(t) r_{E}^{i} r_{E}^{j}+C(t, \mathbf{x})\right]+O\left(c^{-5}\right), \\
X^{a}=\delta_{a i}\left[r_{E}^{i}+\frac{1}{c^{2}}\left(\frac{1}{2} v_{E}^{i} v_{E}^{j} r_{E}^{j}+w_{e x t}\left(\mathbf{x}_{E}\right) r_{E}^{i}+r_{E}^{i} a_{E}^{j} r_{E}^{j}-\frac{1}{2} a_{E}^{i} r_{E}^{2}\right)\right]+O\left(c^{-4}\right),
\end{gathered}
$$

où

$$
\begin{gathered}
\frac{d}{d t} A(t)=\frac{1}{2} v_{E}^{2}+w_{e x t}\left(\mathbf{x}_{E}\right), \\
\frac{d}{d t} B(t)=-\frac{1}{8} v_{E}^{4}-\frac{3}{2} v_{E}^{2} w_{e x t}\left(\mathbf{x}_{E}\right)+4 v_{E}^{i} w_{e x t}^{i}\left(\mathbf{x}_{E}\right)+\frac{1}{2} w_{e x t}^{2}\left(\mathbf{x}_{E}\right), \\
B^{i}(t)=-\frac{1}{2} v_{E}^{2} v_{E}^{i}+4 w_{e x t}^{i}\left(\mathbf{x}_{E}\right)-3 v_{E}^{i} w_{e x t}\left(\mathbf{x}_{E}\right), \\
B^{i j}(t)=-v_{E}^{i} \delta_{a j} Q^{a}+2 \frac{\partial}{\partial x^{j}} w_{e x t}^{i}\left(\mathbf{x}_{E}\right)-v_{E}^{i} \frac{\partial}{\partial x^{j}} w_{e x t}\left(\mathbf{x}_{E}\right)+\frac{1}{2} \delta_{i j} \dot{w}_{e x t}\left(\mathbf{x}_{E}\right), \\
C(t, \mathbf{x})=-\frac{1}{10} r_{E}^{2}\left(\dot{a}_{E}^{i} r_{E}^{i}\right),
\end{gathered}
$$

dans ces formules, $x_{E}^{i}, v_{E}^{i}$, et $a_{E}^{i}$, sont respectivement les composantes des vecteurs position, vitesse et accélération barycentriques de la Terre, le point signifiant la dérivée totale par rapport à $t$, et

$$
Q^{a}=\delta_{a i}\left[\frac{\partial}{\partial x^{i}} w_{e x t}\left(\mathbf{x}_{E}\right)-a_{E}^{i}\right]
$$

Les potentiels extérieurs, $w_{e x t}$ et $w_{e x t}^{i}$, sont donnés par

$$
w_{e x t}=\sum_{A \neq E} w_{A}, \quad w_{e x t}^{i}=\sum_{A \neq E} w_{A}^{i},
$$

où $E$ se rapporte à la Terre et $w_{A}$ et $w_{A}^{i}$ sont déterminés par les expressions de $w$ et $w_{A}^{i}$, les intégrales étant calculées sur le corps A seulement.

\section{Notes}

Il doit être entendu que ces expressions pour $w$ et $w^{i}$ donnent une expression de $g_{00}$

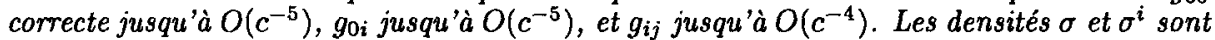
déterminées par les composantes du tenseur impulsion-énergie de la matière composant le système solaire; elles sont données dans les références ci-après. Les exactitudes pour $G_{a b}$ en termes de $c^{-n}$ correspondent à celles de $g_{\mu \nu}$.

Les potentiels extérieurs $W_{e x t}$ et $W_{x t}^{a}$ peuvent être écrits sous la forme suivante :

$$
\begin{aligned}
& W_{e x t}=W_{\text {tidal }}+W_{\text {iner }}, \\
& W_{\text {ext }}^{a}=W_{\text {tidal }}^{a}+W_{\text {iner }}^{a} .
\end{aligned}
$$

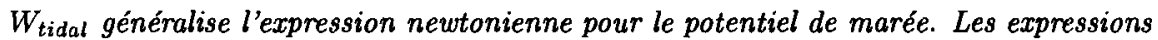
post-newtoniennes de $W_{\text {tidal }}$ et $W_{\text {tidal }}^{a}$ se trouvent dans les références ci-après. Les potentiels $W_{\text {iner }}, W_{\text {iner }}^{a}$ sont les contributions inertielles linéaires en $\mathrm{X}^{a}$. Le premier est déterminé essentiellement par le couplage entre la non-sphéricité de la Terre et le potentiel extérieur. Dans le système de référence céleste géocentrique cinématiquement non tournant, $W_{i n e r}^{a}$ décrit la force de Coriolis produite essentiellement par la précession géodésique.

Enfin, les potentiels gravitationnels locaux $W_{E}$ et $W_{E}^{a}$ de la Terre se rattachent aux potentiels gravitationnels $w_{E}$ et $w_{E}^{i}$ par 


$$
\begin{gathered}
W_{E}(T, \mathbf{X})=w_{E}(t, \mathbf{x})\left(1+\frac{2}{c^{2}} v_{E}^{2}\right)-\frac{4}{c^{2}} v_{E}^{i} w_{E}^{i}(t, \mathbf{x})+O\left(c^{-4}\right), \\
W_{E}^{a}(T, \mathbf{X})=\delta_{a i}\left(w_{E}^{i}(t, \mathbf{x})-v_{E}^{i} w_{E}(t, \mathbf{x})\right)+O\left(c^{-2}\right) .
\end{gathered}
$$

\section{Références}

Brumberg, V.A., Kopeikin, S.M., 1988, Nuovo Cimento $B$,103, 63.

Brumberg, V.A., 1991, Essential Relativistic Celestial Mechanics, Hilger, Bristol.

Damour, T., Soffel, M., Xu, C., Phys.Rev. D 43, 3273 (1991); 45, 1017 (1992); 47, 3124 (1993); 49, 618 (1994).

Klioner, S. A., Voinov, A.V., 1993, Phys Rev. D, 48, 1451.

Kopeikin, S.M., 1989, Celest. Mech., 44, 87.

\section{B1.4 POST-NEWTONIAN POTENTIAL COEFFICIENTS}

The XXIVth International Astronomical Union General Assembly,

\section{Considering}

1. that for many applications in the fields of celestial mechanics and astrometry a suitable parametrisation of the metric potentials (or multipole moments) outside the massive solar system bodies in the form of expansions in terms of potential coefficients are extremely useful, and

2. that physically meaningful post-Newtonian potential coefficients can be derived from the literature,

\section{Recommends}

1. expansion of the post-Newtonian potential of the Earth in the Geocentric Celestial Reference System (GCRS) outside the Earth in the form

$$
W_{E}(T, \mathbf{X})=\frac{G M_{E}}{R}\left[1+\sum_{l=2}^{\infty} \sum_{m=0}^{+l}\left(\frac{R_{E}}{R}\right)^{l} P_{l m}(\cos \theta)\left(C_{l m}^{E}(T) \cos m \phi+S_{l m}^{E}(T) \sin m \phi\right)\right]
$$

here $C_{l m}^{E}$ and $S_{l m}^{E}$ are, to sufficient accuracy, equivalent to the post-Newtonian multipole moments introduced by Damour et al. (Damour et al., Phys. Rev D, 43, 3273, 1991). $\theta$ and $\phi$ are the polar angles corresponding to the spatial coordinates $X^{a}$ of the GCRS and $R=|\mathbf{X}|$, and

2. expression of the vector potential outside the Earth, leading to the well- known Lense-Thirring effect, in terms of the Earth's total angular momentum vector $\mathbf{S}_{E}$ in the form

$$
W_{E}^{a}(T, \mathbf{X})=-\frac{G\left(\mathbf{X} \times \mathbf{S}_{E}\right)^{\mathbf{a}}}{2 R^{3}}
$$




\section{B1.4 COEFFICIENTS POST-NEWTONIENS DU POTENTIEL}

La XXIVème Assemblée générale de l'Union Astronomique Internationale,

\section{Considérant}

1. que pour de nombreuses applications dans le domaine de la mécanique céleste et de l'astrométrie, une paramétrisation des potentiels métriques (ou des moments multipolaires) en dehors des corps massifs du système solaire sous forme de développements en fonction de coefficients du potentiel sont extrêmement utiles,

2. que des coefficients post-newtoniens du potentiel physiquement significatifs peuvent être trouvés dans des publications,

\section{Recommande}

1. de développer le potentiel terrestre post-newtonien dans le système de référence céleste géocentrique (GCRS) en dehors de la Terre sous la forme

$$
W_{E}(T, \mathbf{X})=\frac{G M_{E}}{R}\left[1+\sum_{l=2}^{\infty} \sum_{m=0}^{+l}\left(\frac{R_{E}}{R}\right)^{l} P_{l m}(\cos \theta)\left(C_{l m}^{E}(T) \cos m \phi+S_{l m}^{E}(T) \sin m \phi\right)\right],
$$

Dans cette expression, $C_{l m}^{E}$ et $S_{l m}^{E}$ sont, avec une précision suffisante, équivalents aux moments multipolaires post-newtoniens introduits par Damour et al. (Phys. Rev. D, 43, $3273,1991)$. $\theta$ et $\phi$ sont les angles polaires correspondant aux coordonnées spatiales $X^{a} \mathrm{du}$ GCRS et $R=|\mathbf{X}|$, et

2. d'exprimer le potentiel vecteur en dehors de la Terre, donnant l'effet bien connu de Lense-Thirring en fonction du moment angulaire total de la Terre $\mathbf{S}_{E}$, sous la forme

$$
W_{E}^{a}(T, \mathbf{X})=-\frac{G\left(\mathbf{X} \times \mathbf{S}_{E}\right)^{a}}{2 R^{3}}
$$

\section{B1.5 EXTENDED RELATIVISTIC FRAMEWORK FOR TIME TRANSFORMATIONS AND REALISATION OF COORDINATE TIMES IN THE SOLAR SYSTEM}

The XXIVth International Astronomical Union General Assembly,

\section{Considering}

1. that the Resolution A4 of the XXIst General Assembly (1991) has defined systems of space-time coordinates for the solar system (Barycentric Reference System) and for the Earth (Geocentric Reference System), within the framework of General Relativity,

2. that Resolution B1.3 entitled "Definition of Barycentric Celestial Reference System and Geocentric Celestial Reference System" has renamed these systems the Barycentric Celestial Reference System (BCRS) and the Geocentric Celestial Reference System (GCRS), respectively, and has specified a general framework for expressing their metric tensor and 
defining coordinate transformations at the first post-Newtonian level,

3. that, based on the anticipated performance of atomic clocks, future time and frequency measurements will require practical application of this framework in the BCRS,

4. that theoretical work requiring such expansions has already been performed,

\section{Recommends}

that for applications that concern time transformations and realisation of coordinate times within the solar system, Resolution B1.3 be applied as follows :

1. the metric tensor be expressed as

$$
\begin{gathered}
g_{00}=-\left[1-\frac{2}{c^{2}}\left(w_{0}(t, \mathbf{x})+\left(w_{L}(t, \mathbf{x})\right)+\frac{2}{c^{4}}\left(w_{0}^{2}(t, \mathbf{x})+\Delta(t, \mathbf{x})\right)\right]\right. \\
g_{0 i}=-\frac{4}{c^{3}} w^{i}(t, \mathbf{x}), \\
g_{i j}=\delta_{i j}\left(1+\frac{2}{c^{2}} w_{0}(t, \mathbf{x})\right),
\end{gathered}
$$

where ( $t \equiv$ Barycentric Coordinate Time (TCB), $\mathbf{x})$ are the barycentric coordinates, $w_{0}=G \sum_{A} M_{A} / r_{A}$, with the summation carried out over all solar system bodies $\mathrm{A}$, $\mathbf{r}_{A}=\mathbf{x}-\mathbf{x}_{A}, \mathbf{x}_{A}$ are the coordinates of the centre of mass of body $A, r_{A}=\left|\mathbf{r}_{A}\right|$, and where $w_{L}$ contains the expansion in terms of multipole moments [see their definition in the Resolution B1.4 entitled "Post-Newtonian Potential Coefficients"] required for each body; the vector potential $w^{i}(t, \mathbf{x})=\sum_{A} w_{A}^{i}(t, \mathbf{x})$ and the function $\Delta(t, \mathbf{x})=\sum_{A} \Delta_{A}(t, \mathbf{x})$ are given in note 2,

2. the relation between TCB and Geocentric Coordinate Time (TCG) can be expressed to sufficient accuracy by

$$
\begin{aligned}
\text { TCB }-\mathrm{TCG} & =c^{-2}\left[\int_{t_{\theta}}^{t}\left(\frac{v_{E}^{2}}{2}+w_{0 e x t}\left(\mathbf{x}_{E}\right)\right) d t+v_{E}^{i} r_{E}^{i}\right] \\
-c^{-4}\left[\int_{t_{0}}^{t}\left(-\frac{1}{8} v_{E}^{4}-\frac{3}{2} v_{E}^{2} w_{0 e x t}\left(\mathbf{x}_{E}\right)+4 v_{E}^{i} w_{e x t}^{i}\left(\mathbf{x}_{E}\right)+\frac{1}{2} w_{0 e x t}^{2}\left(\mathbf{x}_{E}\right)\right) d t\right. & \left.-\left(3 w_{0 e x t}\left(\mathbf{x}_{E}\right)+\frac{v_{E}^{2}}{2}\right) v_{E}^{i} r_{E}^{i}\right]
\end{aligned}
$$

where $v_{E}$ is the barycentric velocity of the Earth and where the index ext refers to summation over all bodies except the Earth.

\section{Notes}

1. This formulation will provide an uncertainty not larger than $5 \times 10^{-18}$ in rate and, for quasi-periodic terms, not larger than $5 \times 10^{-18}$ in rate amplitude and $0.2 \mathrm{ps}$ in phase amplitude, for locations farther than a few solar radii from the Sun. The same uncertainty also applies to the transformation between TCB and TCG for locations within $50000 \mathrm{~km}$ of the Earth. Uncertainties in the values of astronomical quantities may induce larger errors in the formulae. 
2. Within the above mentioned uncertainties, it is sufficient to express the vector potential $w_{A}^{i}(t, \mathbf{x})$ of body $A$ as

$$
w_{A}^{i}(t, \mathbf{x})=G\left[\frac{-\left(\mathbf{r}_{A} \times \mathbf{S}_{A}\right)^{i}}{2 r_{A}^{3}}+\frac{M_{A} v_{A}^{i}}{r_{A}}\right]
$$

where $\mathbf{S}_{A}$ is the total angular momentum of body $A$ and $v_{A}^{i}$ are the components of the barycentric coordinate velocity of body $A$. As for the function $\Delta_{A}(t, \mathbf{x})$, it is sufficient to express it as

$$
\Delta_{A}(t, \mathbf{x})=\frac{G M_{A}}{r_{A}}\left[-2 v_{A}^{2}+\sum_{B \neq A} \frac{G M_{B}}{r_{B A}}+\frac{1}{2}\left(\frac{\left(r_{A}^{k} v_{A}^{k}\right)^{2}}{r_{A}^{2}}+r_{A}^{k} a_{A}^{k}\right)\right]+\frac{2 G v_{A}^{k}\left(\mathbf{r}_{A} \times \mathbf{S}_{A}\right)^{\mathbf{k}}}{r_{A}^{g}}
$$

where $\mathbf{r}_{B A}=\left|\mathbf{x}_{B}-\mathbf{x}_{A}\right|$ and $a_{A}^{k}$ is the barycentric coordinate acceleration of body $A$. In these formulae, the terms in $S_{A}$ are needed only for Jupiter $\left(S \approx 6.9 \times 10^{38} \mathrm{~m}^{2} \mathrm{~s}^{-1} \mathrm{~kg}\right)$ and Saturn $\left(S \approx 1.4 \times 10^{38} \mathrm{~m}^{2} \mathrm{~s}^{-1} \mathrm{~kg}\right)$, in the immediate vicinity of these planets.

3. Because the present Recommendation provides an extension of the IAU 1991 recommendations valid at the full first post-Newtonian level, the constants $L_{C}$ and $L_{B}$ that were introduced in the IAU 1991 recommendations should be defined as $\langle T C G / T C B\rangle=1-L_{C}$ and $\langle T T / T C B\rangle=1-L_{B}$, where TT refers to Terrestrial Time and $\langle>$ refers to a sufficiently long average taken at the geocenter. The most recent estimate of $L_{C}$ is (Irwin, $A$. and Fukushima, T.,1999, Astron. Astroph. 348 , 642-652.)

$$
L_{C}=1.48082686741 \times 10^{-8} \pm 2 \times 10^{-17},
$$

From the Resolution B1.9 on "Redefinition of Terrestrial Time TT", one infers

$$
L_{B}=1.55051976772 \times 10^{-8} \pm 2 \times 10^{-17}
$$

by using the relation $1-L_{B}=\left(1-L_{C}\right)\left(1-L_{G}\right) . L_{G}$ is defined in Resolution B1.9.

Because no unambiguous definition may be provided for $L_{B}$ and $L_{C}$, these constants should not be used in formulating time transformations when it would require knowing their value with an uncertainty of order $1 \times 10^{-16}$ or less.

4. If TCB-TCG is computed using planetary ephemerides which are expressed in terms of a time argument (noted $T_{e p h}$ ) which is close to Barycentric Dynamical Time (TDB), rather than in terms of TCB, the first integral in Recommendation 2 above may be computed as

$$
\int_{t_{0}}^{t}\left(\frac{v_{E}^{2}}{2}+w_{0 e x t}\left(\mathbf{x}_{E}\right)\right) d t=\frac{1}{1-L_{B}} \int_{T e p h 0}^{T e p h}\left(\frac{v_{E}^{2}}{2}+w_{0 e x t}\left(\mathbf{x}_{E}\right)\right) d t .
$$




\section{B1.5 EXTENSION DU CADRE RELATIVISTE POUR LES TRANSFORMATIONS DE TEMPS ET POUR LA REALISATION DES TEMPS-COORDONNEE DANS LE SYSTEME SOLAIRE}

La XXIVème Assemblée générale de l'Union Astronomique Internationale,

\section{Considérant}

1. que la résolution A4 (1991) a défini des systèmes de coordonnées d'espace-temps pour le système solaire (système de référence barycentrique) et pour la Terre (système de référence géocentrique), dans le cadre de la relativité générale,

2. que la résolution B1.3 sur la "définition du système de référence barycentrique et du système de référence géocentrique" donne à ces systèmes respectivement les noms de Système de référence céleste barycentrique (BCRS) et de Système de référence céleste géocentrique (GCRS) et donne le cadre général pour exprimer leurs tenseurs métriques et pour définir les transformations de coordonnées au premier niveau post-newtonien,

3. que, compte-tenu des performances prévues pour les horloges atomiques, les mesures futures de temps et de fréquence exigeront de mettre en pratique ce cadre général dans le BCRS,

4. que les travaux théoriques demandant ces développernents ont dêjà été effectués.

\section{Recommande}

que, pour des applications relatives aux transformations de temps et la construction des temps-coordonnée dans le système solaire, la résolution $\mathrm{B} 1.3$ soit appliquée comme suit :

1. le tenseur métrique soit exprimé sous la forme suivante :

$$
\begin{gathered}
g_{00}=-\left[1-\frac{2}{c^{2}}\left(w_{0}(t, \mathbf{x})+\left(w_{L}(t, \mathbf{x})\right)+\frac{2}{c^{4}}\left(w_{0}^{2}(t, \mathbf{x})+\Delta(t, \mathbf{x})\right)\right],\right. \\
g_{0 i}=-\frac{4}{c^{3}} w^{i}(t, \mathbf{x}), \\
g_{i j}=\delta_{i j}\left(1+\frac{2}{c^{2}} w_{0}(t, \mathbf{x})\right),
\end{gathered}
$$

où $(t \equiv$ Temps-coordonnée barycentrique (TCB), $\mathbf{x})$ sont les coordonnées barycentriques, où $w_{0}=G \sum_{A} M_{A} / r_{A}$, la sommation étant faite sur tous les corps $\mathrm{A}$ du ystème solaire, $\mathbf{r}_{A}=\mathbf{x}-\mathbf{x}_{A}, \mathbf{x}_{A}$ sont les coordonnées du centre de masse du corps $A, r_{A}=\left|\mathbf{r}_{A}\right|$ et où $w_{L}$ contient le développement en terme de moments multipolaires [voir la résolution $\mathrm{B1}$ 4 sur les "coefficients du potentiel post-newtonien"] pour chaque corps; le vecteur potentiel $w^{i}(t, \mathbf{x})=\sum_{A} w_{A}^{i}(t, \mathbf{x})$ et la fonction $\Delta(t, \mathbf{x})=\sum_{A} \Delta_{A}(t, \mathbf{x})$ sont donnés dans la note 2 ,

2. la relation entre TCB et le temps-coordonnée géocentrique (TCG) peut être exprimée avec une précision suffisante par :

$$
\mathrm{TCB}-\mathrm{TCG}=c^{-2}\left[\int_{t_{0}}^{t}\left(\frac{v_{E}^{2}}{2}+w_{0 e x t}\left(\mathbf{x}_{E}\right)\right) d t+v_{E}^{i} r_{E}^{i}\right]
$$




$$
\begin{aligned}
-c^{-4}\left[\int _ { t _ { 0 } } ^ { t } \left(-\frac{1}{8} v_{E}^{4}-\right.\right. & \left.\frac{3}{2} v_{E}^{2} w_{0 e x t}\left(\mathbf{x}_{E}\right)+4 v_{E}^{i} w_{\text {ext }}^{i}\left(\mathbf{x}_{E}\right)+\frac{1}{2} w_{\text {0ext }}^{2}\left(\mathbf{x}_{E}\right)\right) d t \\
& \left.-\left(3 w_{0 e x t}\left(\mathbf{x}_{E}\right)+\frac{v_{E}^{2}}{2}\right) v_{E}^{i} r_{E}^{i}\right]
\end{aligned}
$$

où $v_{E}$ est la vitesse barycentrique de la Terre et où l'indice ext se rapporte à la sommation sur tous les corps sauf la Terre.

\section{Notes}

1. Cette formulation assurera une incertitude ne dépassant pas $5 \times 10^{-18}$ en marche et, pour les termes quasi-périodiques, ne dépassant pas $5 \times 10^{-18}$ en amplitude de marche et 0.2 ps en amplitude de phase, pour des lieux plus éloignés du Soleil que quelques rayons solaires. La même majoration de l'incertitude s'applique aussi à la transformation entre TCB et TCG pour des lieux situés à moins de $50000 \mathrm{~km}$ de la Terre. Les incertitudes dans les valeurs de certaines quantités astronomiques pourraient induire des erreurs plus grandes dans les formules.

2. Dans la limite des incertitudes données ci-dessus, il suffit d'exprimer le potentiel vecteur $w^{i}(t, \mathbf{x}) d u$ corps $A$ par

$$
w_{A}^{i}(t, \mathbf{x})=G\left[\frac{-\left(\mathbf{r}_{A} \times \mathbf{S}_{A}\right)^{i}}{2 r_{A}^{3}}+\frac{M_{A} v_{A}^{i}}{r_{A}}\right],
$$

où $\mathbf{S}_{A}$ est le moment cinétique total du corps $A$ et $v_{A}^{i}$ est la vitesse du corps $A$ en coordonnées barycentriques. En ce qui concerne la fonction $\Delta_{A}(t, \mathbf{x})$, il suffit d'utiliser l'expression

$$
\Delta_{A}(t, \mathbf{x})=\frac{G M_{A}}{r_{A}}\left[-2 v_{A}^{2}+\sum_{B \neq A} \frac{G M_{B}}{r_{B A}}+\frac{1}{2}\left(\frac{\left(r_{A}^{k} v_{A}^{k}\right)^{2}}{r_{A}^{2}}+r_{A}^{k} a_{A}^{k}\right)\right]+\frac{2 G v_{A}^{k}\left(r_{A} \times \mathrm{S}_{A}\right)^{\mathbf{k}}}{r_{A}^{3}}
$$

où $\mathbf{r}_{B A}=\left|\mathbf{x}_{B}-\mathbf{x}_{A}\right|$ et $a_{A}^{k}$ est l'accélération du corps $A$ en coordonnées barycentriques. Dans ces formules, les termes en $\mathbf{S}_{A}$ ne sont nécessaires que pour Jupiter $\left(S \approx 6.9 \times 10^{38} \mathrm{~m}^{2} \mathrm{~s}^{-1} \mathrm{~kg}\right)$ et Saturne $\left(S \approx 1.4 \times 10^{38} \mathrm{~m}^{2} \mathrm{~s}^{-1} \mathrm{~kg}\right)$, dans le voisinage immédiat de ces planètes.

3. En raison du fait que la présente recommandation constitue une extension des recommandations UAI 1991 valable au niveau post-newtonien complet, les constantes $L_{C}$ et $L_{B}$ qui avaient été introduites par les recommandations UAI 1991 doivent être définies comme signifiant $\langle T C G / T C B\rangle=1-L_{C}$ et $\langle T T / T C B\rangle=1-L_{B}$, où $T T$ est le temps terrestre et $\langle>$ se rapporte à une moyenne calculée au centre de la Terre sur une durée suffisamment longue. L'estimation la plus récente de $L_{C}$ est (Irwin et Fukushima, 1999, Astron. Astrophys. 348 , 642-652)

$$
L_{C}=1.48082686741 \times 10^{-8} \pm 2 \times 10^{-17},
$$
relation

De la résolution $B 1.9$, sur la redéfinition du temps terrestre $T T$, et en utilisant la

$$
1-L_{B}=\left(1-L_{C}\right)\left(1-L_{G}\right),
$$

où $L_{G}$ est défini par la résolution $B 1.9$, il résulte que

$$
L_{B}=1.55051976772 \times 10^{-8} \pm 2 \times 10^{-17} .
$$


Comme il n'est pas possible de donner une définition non ambigüe de $L_{B}$ et $L_{C}$, ces constantes ne doivent pas être utilisées pour formuler des transformations de temps lorsqu'il sera nécessaire de connaître leur valeur avec une incertitude de l'ordre de $1 \times 10^{-16}$ ou moins.

4. Si TCB-TCG est calculé à partir d'éphémérides planétaires exprimées en termes d'un argument temps (noté $T_{\text {eph }}$ ) proche du temps dynamique barycentrique (TDB), plutôt qu'en terme du TCB, la première intégrale de la recommandation 2 ci-dessus peut être calculée de la manière suivante :

$$
\int_{t_{0}}^{t}\left(\frac{v_{E}^{2}}{2}+w_{0 e x t}\left(\mathbf{x}_{E}\right)\right) d t=\frac{1}{1-L_{B}} \int_{\text {Tepho }}^{T e p h}\left(\frac{v_{E}^{2}}{2}+w_{0 e x t}\left(\mathbf{x}_{E}\right)\right) d t .
$$

\section{B1.6 IAU PRECESSION-NUTATION MODEL}

The XXIVth International Astronomical Union General Assembly,

\section{Recognising}

1. that the International Astronomical Union and the International Union of Geodesy and Geophysics Working Group (IAU-IUGG WG) on 'Non-rigid Earth Nutation Theory' has met its goals by

a. establishing new high precision rigid Earth nutation series, such as (1) SMART97 of Bretagnon et al., 1998, Astron. Astroph. 329, 329-338; (2) REN2000 of Souchay et al., 1999, Astron. Astroph. Supl. Ser. 135, 111-131; (3) RDAN97 of Roosbeek and Dehant 1999, Celest. Mech. 70, 215-253,

b. completing the comparison of new non-rigid Earth transfer functions for an Earth initially in non-hydrostatic equilibrium, incorporating mantle anelasticity and a Free Core Nutation period in agreement with observations,

c. noting that numerical integration models are not yet ready to incorporate dissipation in the core, and

d. noting the effects of other geophysical and astronomical phenomena that must be modelled, such as ocean and atmospheric tides, that need further development;

2. that, as instructed by IAU Recommendation C1 in 1994, the International Earth Rotation Service (IERS) will publish in the IERS Conventions (2000) a precession-nutation model that matches the observations with a weighted $\mathrm{rms}$ of 0.2 milliarcsecond (mas);

3. that semi-analytical geophysical theories of forced nutation are available which incorporate some or all of the following - anelasticity and electromagnetic couplings at the core-mantle and inner core-outer core boundaries, annual atmospheric tide, geodetic nutation, and ocean tide effects;

4. that ocean tide corrections are necessary at all nutation frequencies; and

5. that empirical models based on a resonance formula without further corrections do also exist. 


\section{Accepts}

the conclusions of the IAU-IUGG WG on Non-rigid Earth Nutation Theory published by Dehant et al., 1999, Celest. Mech.. 72 (4), 245-310 and the recent comparisons between the various possibilities, and

\section{Recommends}

that, beginning on 1 January 2003, the IAU 1976 Precession Model and IAU 1980 Theory of Nutation, be replaced by the precession-nutation model IAU 2000A (MHB2000, based on the transfer functions of Mathews, Herring and Buffett, 2000 - submitted to the Journal of Geophysical Research) for those who need a model at the 0.2 mas level, or its shorter version IAU $2000 \mathrm{~B}$ for those who need a model only at the 1 mas level, together with their associated precession and obliquity rates, and their associated celestial pole offsets at J2000.0, to be published in the IERS Conventions 2000, and

\section{Encourages}

1. the continuation of theoretical developments of non-rigid Earth nutation series,

2. the continuation of VLBI observations to increase the accuracy of the nutation series and the nutation model, and to monitor the unpredictable free core nutation, and

3. the development of new expressions for precession consistent with the IAU $2000 \mathrm{~A}$ model.

\section{B1.6 MODELE DE PRECESSION-NUTATION UAI 2000}

La XXIVème Assemblée générale de l'Union Astronomique Internationale,

\section{Reconnaissant}

1. que le groupe de travail (IAU-IUGG WG) sur la théorie de la nutation de la Terre non rigide de l'Union Astronomique Internationale et de l'Union Internationale de Géodésie et de Géophysique a atteint ses objectifs en

a. établissant de nouvelles séries de nutation de la Terre rigide de haute précision telles que (1) SMART97 de Bretagnon et al., 1998, Astron. Astroph. 329, 329-338; (2) REN2000 de Souchay et al., 1999, Astron. Astroph. Supl. Ser. 135, 111-131; (3) RDAN97 de Roosbeek and Dehant 1999, Celest. Mech. 70, 215-253,

b. ayant achevé la comparaison des nouvelles fonctions de transfert de la Terre non-rigide pour une Terre initialement en équilibre non hydrostatique, incorporant l'inélasticité du manteau et une période de la nutation libre du noyau en accord avec les observations,

c. notant que les modèles utilisant l'intégration numérique ne sont pas encore prêts à incorporer la dissipation dans le noyau, et

d. prenant acte que d'autres effets dus à des phénomènes géophysiques et astronomiques tels que les marées océaniques et atmosphériques doivent être modélisés et doivent faire l'objet de nouveaux travaux;

2. que, suivant les instructions données par la recommandation UAI C1 de 1994, le Service international de la rotation de la Terre (IERS) publiera dans les Conventions IERS 
(2000) un modèle de précession-nutation qui rend compte des observations avec une erreur quadratique moyenne pondérée de 0.2 millième de seconde de degré (mas);

3. qu'il existe des théories géophysiques semi-analytiques de la nutation forcée qui incorporent tout ou partie des phénomènes suivants : inélasticité et couplage électromagnétique aux frontières noyau-manteau et graine-noyau extérieur, la marée atmosphérique annuelle, la nutation géodésique et les effets des marées océaniques;

4. que les corrections de marées océaniques sont nécessaires à toutes les fréquences de la nutation; et

5. que des modèles empiriques basés sur une formule de résonance sans autre correction existent également;

\section{Accepte}

les conclusions de l'IAU-IUGG WG sur la théorie de la nutation de la Terre non rigide publiées par Dehant et al. (1999, Celest. Mech. 72 (4), 245-310) et les comparaisons récentes effectuées entre les diverses possibilités, et,

\section{Recommande}

qu'à compter du ler Janvier 2003, le modèle de précession UAI 1976 et la théorie de la nutation UAI 1980 soient remplacés par le modèle de précession-nutation UAI $2000 \mathrm{~A}$ (MHB 2000, basé sur la fonction de transfert de Mathews, Herring et Buffet, 2000, soumis au Journal of Geophysical Research) pour ceux qui ont besoin d'un niveau de précision de 0.2 mas ou sa version réduite UAI $2000 \mathrm{~B}$ pour ceux qui n'ont besoin que d'un modèle à 1 mas près, avec les termes séculaires de la précession et de l'obliquité qui sont associés à ce modèle, ainsi que le décalage correspondant du pôle à J2000.0 tels qu'ils seront publiés dans les Conventions IERS 2000, et

\section{Encourage}

1. à continuer les recherches théoriques sur les séries de la nutation de la Terre nonrigide,

2. à poursuivre les observations VLBI afin d'accroître l'exactitude des séries et des modèles de nutation, et de mesurer la nutation libre non prévisible du noyau, et

3. à développer de nouvelles expressions de la précession compatible avec le modèle UAI $2000 A$. 


\section{B1.7 DEFINITION OF CELESTIAL INTERMEDIATE POLE}

The XXIVth International Astronomical Union General Assembly,

\section{Noting}

the need for accurate definition of reference systems brought about by unprecedented observational precision, and

\section{Recognising} defined,

1. the need to specify an axis with respect to which the Earth's angle of rotation is

2. that the Celestial Ephemeris Pole (CEP) does not take account of diurnal and higher frequency variations in the Earth's orientation,

\section{Recommends}

1. that the Celestial Intermediate Pole (CIP) be the pole, the motion of which is specified in the Geocentric Celestial Reference System (GCRS, see Resolution B1.3) by motion of the Tisserand mean axis of the Earth with periods greater than two days,

2. that the direction of the CIP at J2000.0 be offset from the direction of the pole of the GCRS in a manner consistent with the IAU 2000A (see Resolution B1.6) precessionnutation model,

3. that the motion of the CIP in the GCRS be realised by the IAU $2000 \mathrm{~A}$ model for precession and forced nutation for periods greater than two days plus additional timedependent corrections provided by the International Earth Rotation Service (IERS) through appropriate astro-geodetic observations,

4. that the motion of the CIP in the International Terrestrial Reference System (ITRS) be provided by the IERS through appropriate astro-geodetic observations and models including high-frequency variations,

5. that for highest precision, corrections to the models for the motion of the CIP in the ITRS may be estimated using procedures specified by the IERS, and

6. that implementation of the CIP be on 1 January 2003 .

\section{Notes}

The forced nutations with periods less than two days are included in the model for the motion of the CIP in the ITRS.

The Tisserand mean axis of the Earth corresponds to the mean surface geographic axis, quoted $B$ axis, in Seidelmann, 1982, Celest. Mech. 27, 79-106.

As a consequence of this resolution, the Celestial Ephemeris Pole is no longer necessary. 


\section{B1.7 DEFINITION DU POLE CELESTE INTERMEDIAIRE}

La XXIVème Assemblée générale de l'Union Astronomique Internationale,

\section{Notant}

la nécessité d'une définition précise des systèmes de référence qu'entraîne l'amélioration sans précédent de la précision des observations, et

\section{Reconnaissant} défini,

1. la nécessité de spécifier l'axe par rapport auquel l'angle de rotation de la Terre est

2. que le Pôle céleste des éphémérides ne permet pas de prendre en compte les variations de l'orientation de la Terre diurnes et de fréquence plus élevée,

\section{Recommande}

1. que le Pôle céleste intermédiaire (CIP) soit le pôle dont le mouvement dans le Système de référence céleste géocentrique (GCRS, voir la résolution $\mathrm{B} 1.3$ ) correspond à celui de l'axe moyen de Tisserand de la. Terre limité aux composantes de périodes supérieures à 2 jours,

2. que la direction du CIP à J2000.0 soit décalée de la direction du pôle du GCRS d'une façon cohérente avec le modèle de précession - nutation IAU 2000A (voir la résolution B1.6),

3. que le mouvement du CIP dans le GCRS soit réalisé par le modèle IAU $2000 \mathrm{~A}$ de la précession et de la nutation forcée pour les périodes supérieures à deux jours corrigée des corrections additionnelles dépendant du temps fournies par le Service international de la rotation terrestre (IERS) à partir des observations astro-géodésiques appropriées,

4. que le mouvement du CIP dans le Système de référence international terrestre (ITRS) soit fourni par l'IERS à partir d'observations astro-géodésiques appropriées et des modèles incluant des variations à haute fréquence,

5. que, pour obtenir une plus grande précision, les corrections aux modèles du mouvement du CIP dans l'ITRS peuvent être estimées en utilisant des procédures spécifiées par l'IERS, et

6. que la mise en œuvre du CIP ait lieu le ler Janvier 2003.

\section{Notes}

Les nutations forcées ayant des périodes inférieures à deux jours sont incluses dans le modèle du mouvement du CIP dans l'ITRS.

L'axe moyen de Tisserand de la Terre correspond à l'axe géographique moyen appelé axe B par Seidelmann, 1982 (Celest. Mech. 27, 79-106). nécessaire.

La conséquence de cette résolution, est que le Pôle céleste des éphémérides n'est plus 


\section{B1.8 DEFINITION AND USE OF CELESTIAL AND TERRESTRIAL EPHEMERIS ORIGINS}

The XXIVth International Astronomical Union General Assembly,

\section{Recognising}

1. the need for reference system definitions suitable for modern realisations of the conventional reference systems and consistent with observational precision,

2. the need for a rigorous definition of sidereal rotation of the Earth,

3. the desirability of describing the rotation of the Earth independently from its orbital motion, and

\section{Noting}

that the use of the "non-rotating origin" (Guinot, 1979) on the moving equator fulfills the above conditions and allows for a definition of UT1 which is insensitive to changes in models for precession and nutation at the microarcsecond level,

\section{Recommends}

1. the use of the "non-rotating origin" in the Geocentric Celestial Reference System ((GCRS) and that this point be designated as the Celestial Ephemeris Origin (CEO) on the equator of the Celestial Intermediate Pole (CIP),

2. the use of the "non-rotating origin" in the International Terrestrial Reference System (ITRS) and that this point be designated as the Terrestrial Ephemeris Origin (TEO) on the equator of the CIP,

3. that UT1 be linearly proportional to the Earth Rotation Angle defined as the angle measured along the equator of the CIP between the unit vectors directed toward the CEO and the TEO,

4. that the transformation between the ITRS and GCRS be specified by the position of the CIP in the GCRS, the position of the CIP in the ITRS, and the Earth Rotation Angle,

5. that the International Earth Rotation Service (IERS) take steps to implement this by 1 January 2003 , and

6. that the IERS will continue to provide users with data and algorithms for the conventional transformations.

\section{Note}

The position of the CEO can be computed from the IAU 2000A model for precession and nutation of the CIP and from the current values of the offset of the CIP from the pole of the ICRF at J2000.0 using the development provided by Capitaine et al. (2000).

The position of the TEO is only slightly dependent on polar motion and can be extrapolated as done by Capitaine et al. (2000) using the IERS data. 
The linear relationship between the Earth's rotation angle $\theta$ and UT1 should ensure the continuity in phase and rate of UT1 with the value obtained by the conventional relationship between Greenwich Mean Sidereal Time (GMST) and UT1. This is accomplished by the following relationship :

$$
\begin{gathered}
\theta(U T 1)=2 \pi(0.7790572732640+1.00273781191135448 \times(\text { Julian UT1 date } \\
-2451545.0))
\end{gathered}
$$

\section{References}

Guinot, B., 1979, in D.D. McCarthy and J.D. Pilkington (eds.), Time and the Earth's Rotation, D. Reidel Publ. 7-18. 405.

Capitaine, N., Guinot, B. and Mc Carthy, D. D., 2000, Astron. Astrophys., 335, 398-

\section{B1.8 DEFINITION ET UTILISATION DES ORIGINES CELESTE ET TERRESTRE DES EPHEMERIDES}

La XXIVème Assemblée générale de l'Union Astronomique Internationale,

\section{Reconnaissant}

1. le besoin d'avoir, pour les systèmes de référence, des définitions convenant aux réalisations modernes des systèmes de référence conventionnels et cohérents avec la précision des observations,

2. la nécessité d'une définition rigoureuse de la rotation sidérale de la Terre,

3. les avantages qu'il y a de décrire la rotation de la Terre indépendamment de son mouvement orbital, et

\section{Notant}

que l'utilisation de "l'origine non tournante" (Guinot, 1979) sur l'équateur mobile remplit ces conditions et permet de définir l'UT1 de telle façon qu'il soit insensible à des changements de modèle de précession et de nutation au niveau de la microseconde de degré,

\section{Recommande}

1. l'usage de "l'origine non tournante" pour le Système de référence céleste géocentrique GCRS sur l'équateur du pôle céleste intermédiaire (CIP), ce point étant appelé origine céleste des éphémérides (CEO),

2. l'usage de "l'origine non tournante" pour le système de référence international terrestre (ITRS) sur l'équateur du CIP, ce point étant appelé origine terrestre des éphémérides (TEO),

3. que l'UT1 soit linéairement proportionnel à l'angle de rotation de la Terre, défini comme étant l'angle mesuré le long de l'équateur du CIP entre les vecteurs unitaires dirigés vers le CEO et le TEO,

4. que la transformation entre l'ITRS et le GCRS soit définie par la position du pôle céleste intermédiaire (CIP) dans le système CGRS, la position du CIP dans l'ITRS et l'angle de rotation de la Terre,

5. que le Service International de la rotation de la Terre (IERS) prenne les mesures utiles pour mettre ceci en cuvre le ler Janvier 2003, et 
6. que l'IERS continue de fournir aux usagers les données et les algorithmes pour les transformations conventionnelles.

Note

La position du CEO peut être calculée à partir du modèle UAI $2000 \mathrm{~A}$ de précession - nutation, du CIP et des valeurs du décalage du CIP par rapport au pôle de l'ICRF à J2000.0 en utilisant le développement donné par Capitaine et al. (2000).

La position du TEO ne dépend que très peu du mouvement du pôle et peut être extrapolée ainsi que l'ont fait Capitaine et al. (2000) en utilisant les données de l'IERS.

La relation linéaire entre l'angle de rotation de la Terre $\theta$ et l'UT1 doit permettre d'assurer la continuité en phase et en terme linéaire de l'UT1 avec la valeur obtenue par la relation conventionnelle entre le temps sidéral du méridien de Greenwich et l'UT1. Ceci est réalisé par la relation suivante :

$$
\begin{gathered}
\theta(U T 1)=2 \pi(0.7790572732640+1.00273781191135448 \times(J \text { ulian UT1 date } \\
-2451545.0))
\end{gathered}
$$

\section{Références}

Guinot, B., 1979, in D.D. McCarthy and J.D. Pilkington (eds.), Time and the Earth's Rotation, D. Reidel Publ. 7-18.

Capitaine, N., Guinot, B. and Mc Carthy, D. D., 2000, Astron. Astrophys., 335, 398405.

\section{B1.9 RE-DEFINITION OF TERRESTRIAL TIME TT}

The XXIVth International Astronomical Union General Assembly,

\section{Considering}

1. that IAU Resolution A4 (1991) of the XXIth General Assembly has defined Terrestrial Time (TT) in its Recommendation 4,

2. that the intricacy and temporal changes inherent to the definition and realisation of the geoid are a source of uncertainty in the definition and realisation of $\mathrm{TT}$, which may become, in the near future, the dominant source of uncertainty in realising TT from atomic clocks,

\section{Recommends}

that TT be a time scale differing from TCG by a constant rate : $d T T / d T C G=1-L_{G}$, where $L_{G}=6.969290134 \times 10^{-10}$ is a defining constant,

\section{Note}

$L_{G}$ was defined by the IAU Resolution A4 (1991) in its Recommendation \& as equal to $U_{G} / c^{2}$ where $U_{G}$ is the geopotential at the geoid. $L_{G}$ is now used as a defining constant. 


\section{B1.9 REDEFINITION DU TEMPS TERRESTRE TT}

La XXIVème Assemblée générale de l'Union Astronomique Internationale,

\section{Considérant}

1. que la résolution A4 (1991) de la XXIème Assemblée générale de l'UAI a défini le temps terrestre (TT) dans sa recommandation 4,

2. que la complexité et les changements temporels inhérents à la définition et à la réalisation du géoïde sont des sources d'incertitude dans la définition et la réalisation du TT, qui peuvent devenir, dans un avenir proche, les sources dominantes d'incertitude dans le réalisation du TT à l'aide d'horloges atomiques,

\section{Recommande}

que le temps terrestre (TT) soit une échelle de temps qui differe du temps- coordonnée géocentrique (TCG) par une valeur constante de la dérivée $d T T / d T C G=1-L_{G}$, où

$L_{G}=6.969290134 \times 10^{-10}$ est une constante de définition.

Note

$L_{G}$ a été défini par la résolution $A 4$ (1991) de l'UAI dans sa recommandation \& comme étant égale à $U_{G} / c^{2}$ où $U_{G}$ est le géopotentiel sur le géoüde. $L_{G}$ est maintenant utilisé comme constante de définition.

\section{B2 COORDINATED UNIVERSAL TIME}

The XXIVth International Astronomical Union General Assembly,

\section{Recognising}

1. that the definition of Coordinated Universal Time (UTC) relies on the astronomical observation of the UT1 time scale in order to introduce leap seconds,

2. that the unpredictability of leap seconds affects modern communication and navigation systems,

3. that astronomical observations provide an accurate estimate of the secular deceleration of the Earth's rate of rotation,

\section{Recommends}

1. that the IAU establish a working group reporting to Division I at the General Assembly in 2003 to consider the redefinition of UTC,

2. that this study discuss whether there is a requirement for leap seconds, the possibility of inserting leap seconds at pre-determined intervals, and the tolerance limits for UT1-UTC, and

3. that this study be undertaken in cooperation with the appropriate groups of the International Union of Radio Science (URSI), the International Telecommunications Union (ITU-R), the International Bureau for Weights and Measures (BIPM), the International Earth Rotation Service (IERS), and relevant navigational agencies. 


\section{B2 TEMPS UNIVERSEL COORDONNE}

La XXIVème Assemblée générale de l'Union Astronomique Internationale,

\section{reconnaissant}

1. que la définition du Temps universel coordonné (UTC) repose sur les observations astronomiques de l'échelle de temps UT1 afin d'introduire les secondes intercalaires,

2. que l'imprévisibilité des secondes intercalaires affecte les systèmes modernes de communication et de navigation,

3. que les observations astronomiques fournissent une estimation précise de la décélération de la vitesse de rotation de la Terre,

\section{Recommande}

1. que l'UAI mette en place un groupe de travail pour examiner une éventuelle redéfinition de l'UTC et rendre compte à la Division 1 lors de l'Assemblée générale de 2003 ,

2. que ce groupe de travail discute de la nécessité d'avoir des secondes intercalaires, la possibilité d'insérer des secondes intercalaires à des intervalles prédéterminés et les limites de tolérance pour UT1-UTC, et

3. que cette étude soit entreprise en coopération avec les groupes compétents de l'Union radio-scientifique internationale (URSI), l'Union internationale des télécommunications (ITU$\mathrm{R}$ ), le Bureau international des poids et mesures (BIPM), le Service international de la rotation terrestre (IERS) et les agences de navigation appropriées.

\section{B3 SAFEGUARDING THE INFORMATION IN PHOTOGRAPHIC PLATES}

The XXIVth International Astronomical Union General Assembly,

\section{Consequent upon}

its recommendation C13 (1991) of the XXIst General Assembly to create accessible archives of the large quantities of observational material collected during the 20th century and currently stored on photographic plates,

\section{Recognising}

that unless action is taken this unique historical record of astronomical phenomena will be lost to future generations of astronomers,

\section{Considering}

the important efforts made by the Working Groups on (i) Sky Surveys, (ii) Carte du Ciel plates, and (iii) Spectroscopic Data Archives, as well as by the Centre for European Plates recently launched at the Royal Observatory of Belgium, in locating and cataloguing plates, in defining the tools needed to safeguard them, and in negotiating the means to preserve their recorded informations in digital form in the public domain, 


\section{Realising}

that the cataloguing, storage and safeguarding of the photographic plates is an important aspect for the implementation of the possible future digitalisation processes needed for selective media transfer of high quality data,

\section{Recommends}

the transfer of the historic observations onto modern media by digital techniques, which will provide worldwide access to the data so as to benefit astronomical research in a way that is well matched to the tools of the researcher in the future.

\section{B3 SAUVEGARDE DES DONNEEES DES OBSERVATIONS PHOTOGRAPHIQUES}

La XXIVème Assemblée générale de l'Union Astronomique Internationale,

Conformément à la recommandation C13 de la XXIème Assemblée générale de l'UAI (1991) de créer des archives accessibles des grandes quantités observationnelles amassées au cours du 20ème siècle et gardées actuellement sur des clichés photographiques,

\section{Reconnaissant que}

si des actions ne sont pas prises, ces archives historiques uniques des phénomènes astronomiques seront perdues pour les futures générations d'astronomes,

\section{Considérant}

les efforts importants déployés par les Groupes de travail (1) pour la surveillance du Ciel, (2) pour les clichés de la Carte du Ciel et (3) pour les archives des données spectroscopiques, ainsi que par le Centre pour les clichés européens récemment créé à l'Observatoire Royal de Belgique, pour trouver et cataloguer ces clichés, pour définir les outils nécessaires pour assurer leur sauvegarde et pour négocier les moyens de préserver les informations qu'ils contiennent sous forme digitale accessible au public, et

\section{Reconnaissant}

que le recensement, l'archivage et la sauvegarde des clichés photographiques sont une condition importante pour pouvoir effectuer dans l'avenir la digitalisation nécessaire à un transfert de données de haute qualité sur un support adéquat.

\section{Recommande}

le transfert des observations historiques sur des supports modernes par des techniques digitales, ce qui donnera un accès mondial aux données au bénéfice de la recherche astronomique d'une manière qui convienne aux outils de recherche futurs. 